\title{
Keeping the Chandra Satellite Cool with Python
}

\author{
Tom Aldcroft ${ }^{\ddagger *}$
}

Abstract-The Chandra X-ray Observatory has been providing groundbreaking astronomical data since its launch by NASA in July of 1999. Now starting the second decade of science the Chandra operations team has been using Python to create predictive thermal models of key spacecraft components. These models are being used in the mission planning and command load review process to ensure that the series of planned observations and attitudes for each week will maintain a safe thermal environment. Speaking from my perspective as a scientist working to create and calibrate the models, I will discuss the process and the key off-the-shelf tools that made it all possible. This includes fitting many-parameter models with the Sherpa package, parallel computation with mpi4py/MPICH2, large table manipulations with pytables/HDF5, and of course fast array math with NumPy.

Index Terms-telescope, NASA, MPI, astronomy, control

\section{Motivation}

This paper describes the use of off-the-shelf tools in Python to tackle a relatively challenging engineering problem facing the Chandra X-ray Observatory satellite [CHANDRA]. While presenting no fundamentally new algorithms or packages, the goal here is to take this as a case study for understanding how scientists and engineers can make Python the foundation of their analysis toolkit.

\section{Chandra}

The Chandra satellite was launched in July of 1999 as one of NASA's four "Great Observatories". This satellite can be compared in size and scope to the Hubble Space Telescope except that it views the universe in X-rays instead of optical or UV light. Some people will argue that the universe is a much more exciting place when viewed with X-ray photons, for then the sky comes alive with black holes, supernova explosions, massive galaxy clusters, pulsars, and many other highly energetic phenomena.

Early in the mission it became apparent that temperatures on the spacecraft, particularly on the side which normally faces the Sun, were increasing at a rate well above pre-launch predictions. It turned out that the ionizing particle radiation environment was higher than expected and that it was degrading the silverized teflon insulation which wraps much of the spacecraft. Since this time the constraint of keeping spacecraft components within safe operating temperatures has been a major driver in operations and schedule

\footnotetext{
* Corresponding author: aldcroft@head.cfa.harvard.edu

¥ Smithsonian Astrophysical Observatory
}

Copyright $\odot 2010$ Tom Aldcroft. This is an open-access article distributed under the terms of the Creative Commons Attribution License, which permits unrestricted use, distribution, and reproduction in any medium, provided the original author and source are credited.

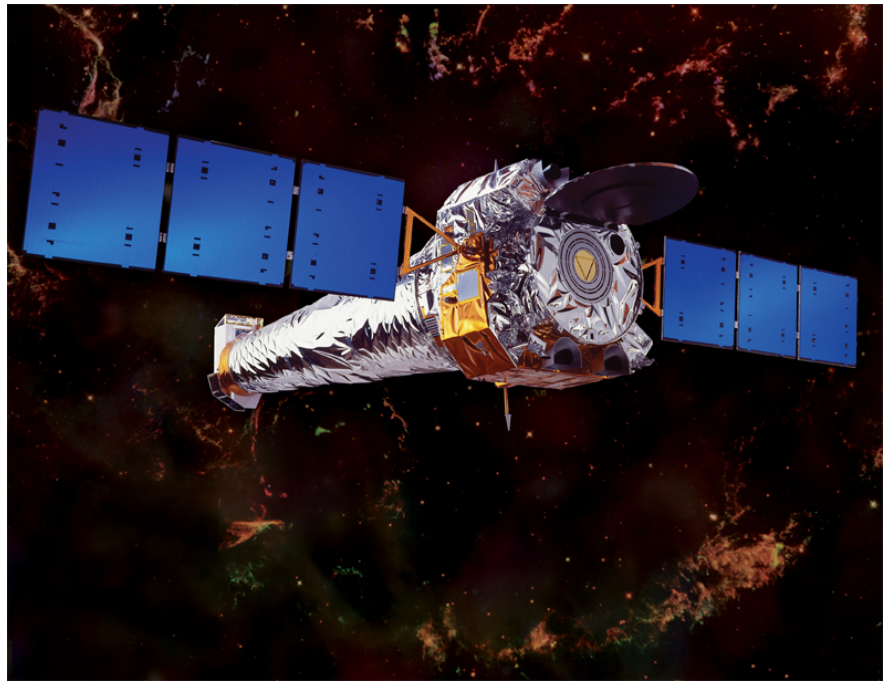

Fig. 1: Artist's rendering of the Chandra X-ray satellite. The silverized teflon which wraps the spacecraft has degraded so it is now far less reflective than shown.

planning. Note that Chandra is in a high elliptical orbit, so unlike Hubble no repair is possible.

Different parts of the spacecraft are heated at different pitch angles (the angle that the telescope boresight axis makes to the sun line). This is shown in Figure 2 which presents a side view of Chandra along with the subsystems that are sensitive over different pitch ranges. Temperatures can be maintained within limits by ping-ponging to different attitudes, never letting one part get too hot. Thus in order to plan the weekly observing schedule a few simple models were created to predict temperatures or otherwise constrain the observing duration at certain attitudes.

As the constraints became more significant a need developed to improve the models in order to maintain the highest scientific output without endangering the spacecraft. In response, about three years ago the CXC Science Operations Team (SOT, scientists closely involved in satellite operations) and the engineers of the Flight Operations Team (FOT) formed a joint working group to study thermal issues and to develop higher fidelity thermal models. This paper discusses one facet of the results coming out of the thermal modeling working group.

Early in the process the author chose Python as the programming language for supporting this effort. Around this time NumPy had emerged as a strong (and consolidated) numeric array manipulation tool for Python. Adding in IPython, Matplotlib and SciPy provided a development and interactive analysis environment that was ideal for the task. 


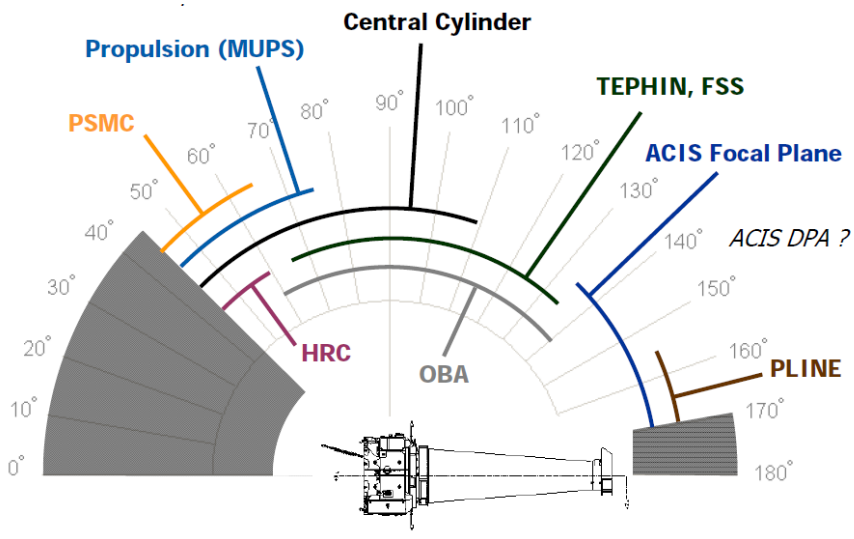

Fig. 2: Thermal constraint regions depending on the pitch angle to the sun line.

\section{Telemetry access}

A key starting point for developing complex thermal models is fast access to thermal and other engineering data from the satellite. The Chandra satellite records a $32 \mathrm{kbit} / \mathrm{s}$ stream of telemetry that contains both the science data and satellite engineering (housekeeping) data. Although the data volumes are meager by modern standards, the tools and data structure used for processing and access were largely designed with mid-1990's era hardware and storage capabilities in mind.

Two standard methods exist for retrieving archival telemetry. Within the Flight Operations Team (FOT) environment at the Operations Control Center the primary tool stores no intermediate products and instead always falls back to the raw telemetry stream. This stream contains over 6000 individual engineering telemetry items (MSIDs) that are interleaved according to different sampling rates and sub-formats. FOT engineers using this system are accustomed to waiting hours or more to retrieve a year of data needed for analysis.

Within the Chandra X-ray Center (CXC) which is responsible for processing the science data, the situation is somewhat better. In this case the "Level-0 decommutation" process is done just once and the results stored in FITS [FITS] files available through an archive server. These files each contain about two hours of data for MSIDs that are related by subsystem (thermal, pointing control, etc) and sampling rate. However, access to a single MSID of interest (e.g. a particular thermistor readout) requires retrieving and unzipping a large amount of uninteresting data.

The solution to this problem was found in the pytables [PYT] package which provides a robust interface to the powerful Hierarchical Data Format [HDF5] library. Pytables/HDF5 is designed to create, update, and access very large data tables with ease. The key here was creating a separate HDF5 table for each MSID which could easily store all the readouts for that MSID for the entire mission. This is especially optimal because many of the MSIDs change infrequently and thus compress very well. HDF5 natively supports an assortment of compression options which makes this a snap. Initially creating the table based on a NumPy data array is simple using the createEArray method to create an extendible homogenous dataset:

filts = tables.Filters (complevel=5, complib='zlib') h5 = tables.openfile (filename, mode=' $\mathrm{w}^{\prime}$, filters=filts) h5shape $=(0)+$, data. shape $[1:]$

h5type = tables.Atom.from_dtype (data.dtype)

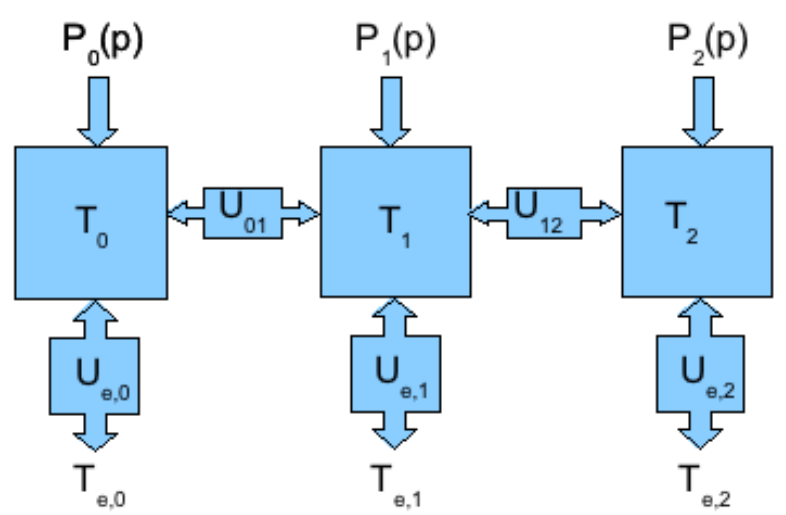

Fig. 3: Schematic diagram of the thermal Chandra thermal model. Boxes $\left(T_{0}, T_{1}, T_{2}\right)$ represent physical nodes on the spacecraft where a thermistor is located. External solar heat input is shown as $P_{i}(p)$, conductances are $U_{i, j}$, and external heat bath temperatures are $T_{e, i}$.

h5.createEArray (h5.root, 'data', h5type, h5shape, title=colname, expectedrows=n_rows) h5.createEArray (h5.root, 'quality', tables.BoolAtom(), $(0)$, title='Quality', expectedrows=n_rows $)$

h5. close ( )

A minor complication seen here is the boolean quality table which accounts for bad or missing telemetry. Once the table has been created it is a simple matter to extend it with new data values after a communication pass:

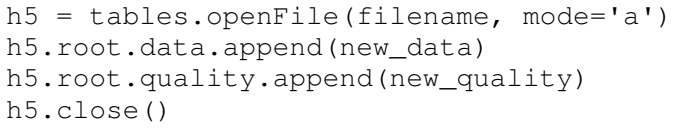

At this time the largest individual tables have about $1.3 \times 10^{9}$ rows (for the highest sampling rate of 4 times per second). The data retrieval speed from this archive of HDF5 tables is approximately $10^{7}$ items per second. This means that typical data retrieval requests can be handled in seconds rather than hours. Such an improvement changes the landscape of questions that can be asked and then answered.

In addition to the data acquisition back-end, a user-friendly front-end was needed to access the telemetry data in the HDF5 archive. A challenge in this regard was that most of the intended user community (FOT engineers) had absolutely no experience with Python. Thus the interface, documentation and examples had to be clear and explicit. The final documentation package included a tutorial covering the telemetry access interface as well as IPython, NumPy, and Matplotlib.

\section{Creating a thermal model}

The thermal model which was developed for modeling Chandra subsystems is illustrated in Figure 3.

Here each of the boxes $\left(T_{0}, T_{1}, T_{2}\right)$ represents a physical node on the spacecraft where a thermistor is located. It is then assumed that each node $i$ has an external heat input $P_{i}(p)$ and has conductances $U_{i, j}$ to other nodes and an external heat bath with temperature $T_{e, i}$. For most models the external heat input is Solar and depends purely on the spacecraft pitch angle with respect to the Sun. In some cases, however, the heat input due to internal electronics is also included. Given these definitions and the nodal connectivity the temperatures can be written in matrix form as a 
simple first order differential equation:

$$
\begin{aligned}
\dot{\mathbf{T}}= & \tilde{\mathbf{A}} \mathbf{T}+\mathbf{b} \\
\mathbf{T}(t)= & \int_{0}^{t} e^{\tilde{\mathbf{A}}(t-u)} \mathbf{b} d u+e^{\tilde{\mathbf{A}} t} \mathbf{T}(0) \\
= & {\left[\mathbf{v}_{1} \mathbf{v}_{2}\right]\left[\begin{array}{cc}
\frac{e^{\lambda_{1} t}-1}{\lambda_{1}} & 0 \\
0 & \frac{e^{\lambda_{2} t}-1}{\lambda_{2}}
\end{array}\right]\left[\mathbf{v}_{1} \mathbf{v}_{2}\right]^{-1} \mathbf{b} } \\
& +\left[\mathbf{v}_{1} \mathbf{v}_{2}\right]\left[\begin{array}{cc}
e^{\lambda_{1} t} & 0 \\
0 & e^{\lambda_{2} t}
\end{array}\right]\left[\mathbf{v}_{1} \mathbf{v}_{2}\right]^{-1} \mathbf{T}(0)
\end{aligned}
$$

Here $\mathbf{T}$ is a vector of node temperatures, $\tilde{\mathbf{A}}$ is the matrix describing the coupling between nodes, $\mathbf{b}$ is a vector describing the heat inputs, $\mathbf{v}_{i}$ and $\lambda_{i}$ are the eigenvectors and eigenvalues of $\tilde{\mathbf{A}}$, and $t$ is time.

The solution can be expressed analytically as long as the model parameters (external heat inputs, conductances) are constant. Most of the time Chandra dwells at a particular attitude and so this is a good assumption during such a dwell. The computational strategy for making a model prediction of temperatures is to identify "states" where the parameters are constant and propagate temperatures from the beginning to the end of the state, then use the end temperatures as the starting point for the next state.

The first implementation of this core model calculation was a literal transcription of the analytic solution for each time step within a state. This was quite inefficient because of repeated creation and computation of intermediate 2-d arrays. A slight modification allowed for adding the time dimension into the arrays and computing all time steps at once with a single expression of NumPy dot products. This resulted in a factor of 10-20 speed increase. Further optimization to avoid repeating certain calculations within inner loops plus caching of results eventually yielded code that is 50 times faster than in the initial literal version. In the end the code takes less than a second to predict a year of temperatures at 5-minute resolution for a 5-node model of the sun-pointed side of the spacecraft.

\section{Fitting the model parameters}

The next step is to tune the model parameters to best fit the existing thermal data for the subsystem of interest. In typical cases there are two to five thermistors whose data are averaged over 5 minute intervals. Up to five years of such data are fit at once.

What is not immediately apparent in the concise matrix formulation $\dot{\mathbf{T}}=\tilde{\mathbf{A}} \mathbf{T}+\mathbf{b}$ of the thermal model is that it contains a lot of free parameters. In addition to the conductances and external heat bath temperatures, the external Solar power input for each node is complicated. First it is a function of the pitch angle with respect to the Sun, but it also has an annual variation term (due to the elliptical orbit) as well as a long-term change due to the continued slow degradation of the protective insulation. All this needs to be fit in order to predict temperature profiles at any time, including years in advance. One key 5-node model being used in planning operations has a total of 80 free parameters. All of those parameters need to calibrated using at least 5 years of existing thermal data to train the model.

Two immediate objections can be raised. First, that with so many free parameters one can fit almost anything. In a sense for this application that is just fine, as long as the resultant model has stable predictive power beyond the time range over which it is calibrated. But at a more fundamental level experience has shown that it is simply not true that the complex and coupled time-dependent behavior of temperatures on the spacecraft can

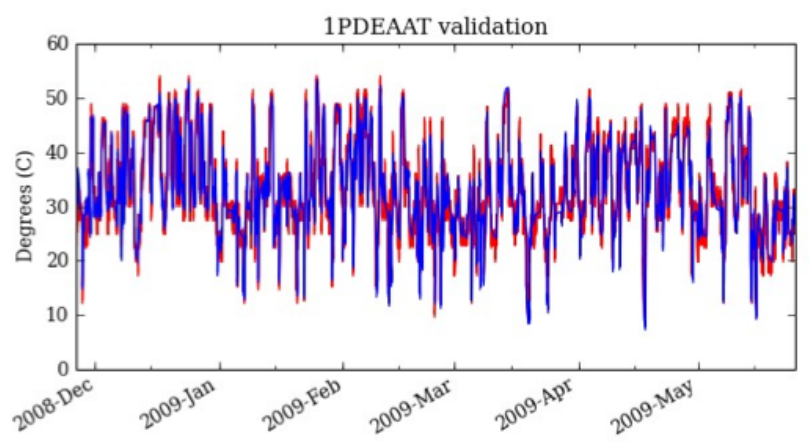

Fig. 4: Long-term comparison of the actual spacecraft thermistor data (red) with the model prediction (blue). This span of data is used for fitting the model coefficients.

necessarily be captured by any model with a large number of parameters.

The second objection is that fitting for so many parameters is bound for failure. However, what makes this problem tractable is that many of the parameters are only loosely coupled. This makes it possible to selectively fit for subsets of the parameters and iteratively home in on a reasonable global set of parameters. Unlike many problems in parameter estimation where the derived parameter values and uncertainties are the primary goal, in this case it is the model prediction that matters.

The Sherpa [SHP] package is used to handle the actual optimization of parameters to achieve the best model fit to the data. Sherpa is a modeling and fitting application for Python that contains a powerful language for combining simple models into complex expressions that can be fit to the data using a variety of statistics and optimization methods. It is easily extendible to include user models, statistics and optimization methods. For this application the key feature is a robust implementation of the Nelder-Mead (aka Simplex) optimization method that is able to handle many free parameters. Sherpa provides within the model language a natural way of manipulating and linking model parameters using Python expressions.

The result of the fitting process is a calibrated thermal model that can be used to accurately predict the system temperatures given the planned sequence of maneuvers and instrument configurations. Figure 4 shows an example of the data for one thermistor "1PDEAAT" in red with the model prediction in blue.

Figure 5 now shows the post-facto model prediction (blue) for a two-week period of data (red) that is outside the calibration time range. Most of the features are well reproduced and the distribution of residuals is roughly gaussian.

\section{Parallelization of fitting}

Despite the good model calculation performance with vectorized NumPy, fitting for 5 years of data and dozens of parameters can benefit from the further speed increase of parallelization. This is particularly helpful for the exploratory phase of developing a new model and getting the parameters in the right ball park.

The thermal models being discussed here can easily be parallelized by splitting into independent time segments. There is a slight issue with the starting conditions for each segment, but there are straightforward ways to finesse this problem. In the context of a fitting application a master-worker architecture works well. Here the master is responsible for controlling the fit 


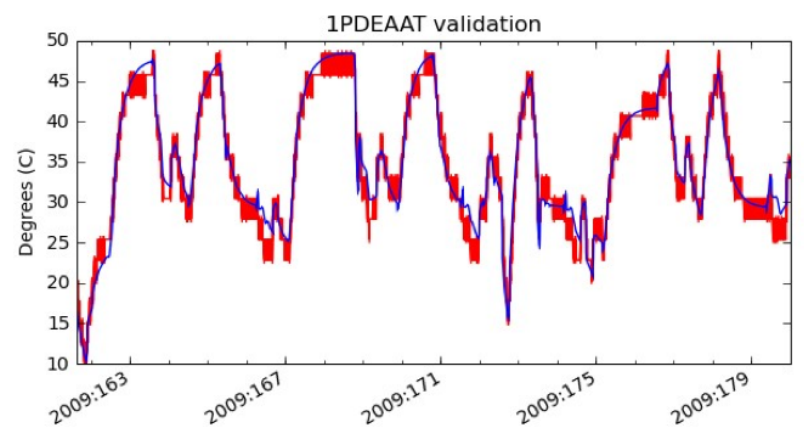

Fig. 5: Detailed comparison of the actual spacecraft thermistor data (red) with the model prediction (blue). The thermistor is located within the power-supply box for one of the main science instruments.

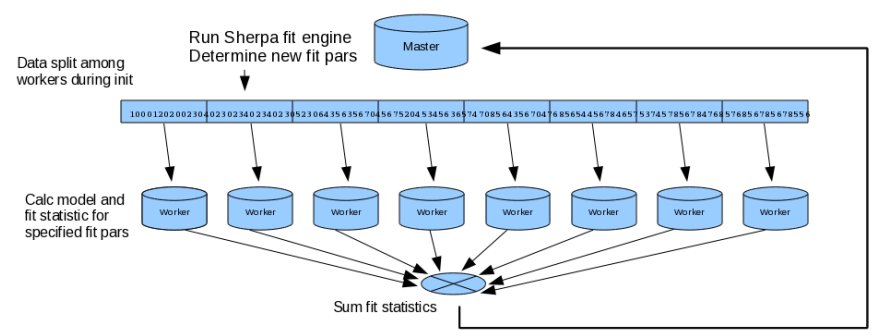

Fig. 6: Schematic illustration of parallelizing the fitting process by breaking the data and model generation into smaller time slices.

optimization process while each of the workers takes care of all model computations for a particular time segment. The worker is initially sent the time range and model definition and it is then responsible for retrieving the appropriate telemetry data. After initialization the model parameters for each fit iteration are sent and the worker computes the model and $C h i^{2}$ fit statistic. All of the individual $C h i^{2}$ values are then summed. In this way the communication overhead between master and workers is minimal. Figure 6 illustrates the process.

The actual job of handling the interprocess communication and job creation is done with the mpi4py [MPI4PY] package using the MPICH2 [MPICH2] library. As is often the case, the choice of these particular packages over other similar ones was driven by the depth of documentation, availability of relevant looking examples, and ease of installation. Starting with no previous experience with distributed computing, a working prototype of the parallel fitting code was created in less than a day. This is a testament largely to the quality of documentation.

As for computing resources, our division within SAO is perhaps like other academic science institutes with a collection of similarly configured linux machines on a local network. These are often available off-hours for "borrowing" CPU cycles with consent of the primary user. A more formal arrangement (for instance using an application like Condor for distributed job scheduling) has been in consideration but not yet adopted. For this application up to twelve 4-core machines were used. Dynamic worker creation was supported by first starting up mpd servers on the target hosts (from file mpd.hosts) with a command like the following:

mpdboot --totalnum=12 --file=mpd.hosts --maxbranch=12

An abridged version of three key functions in the main parallel fitting code is shown below. These functions support communication with and control of the workers:

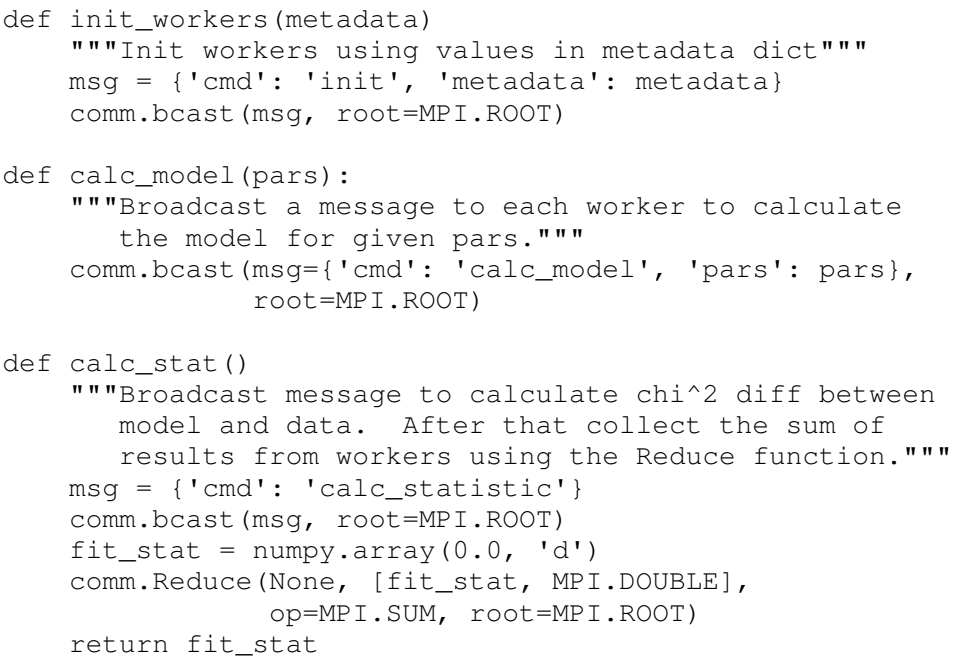

After defining the above functions the main processing code first uses the MPI Spawn method to dynamically create the desired number of worker instances via the previously created mpd servers. Then the workers receive an initialization command with the start and stop date of the data being used in fitting. The Sherpa user model and fit statistic are configured, and finally the Sherpa fit command is executed:

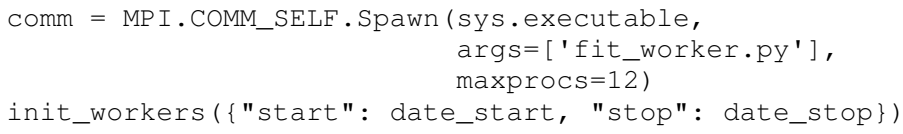

The fit_worker.py code is likewise straightforward. First get a communication object to receive messages, then simply wait for messages with the expected commands. The init command calls the get_data() function that gets the appropriate data given the metadata values and the rank of this worker within the ensemble of size workers.

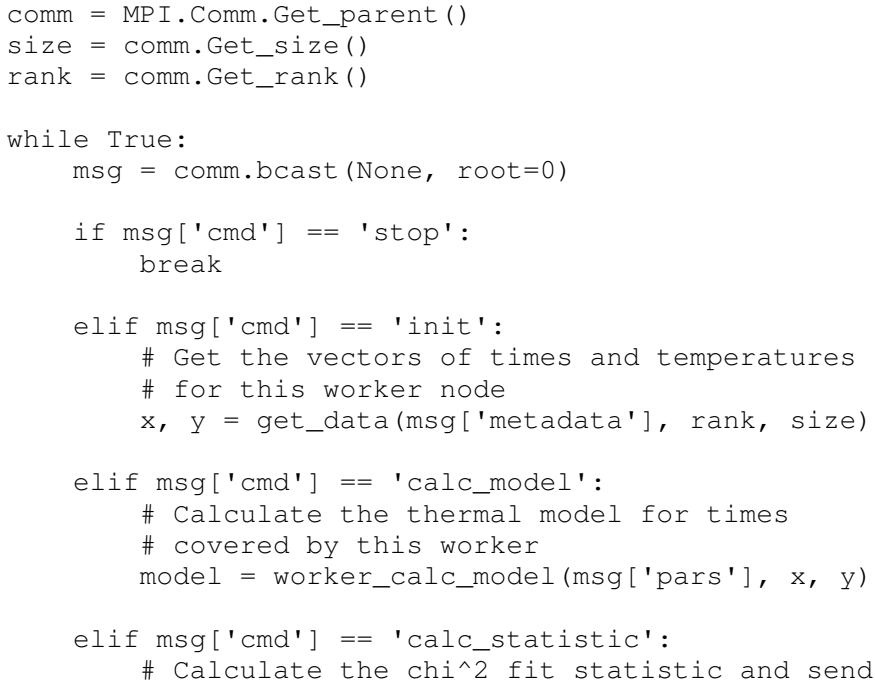


comm. Disconnect ()

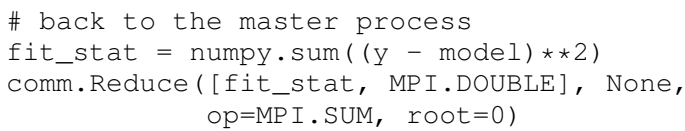

\section{Putting it to work}

Using the techniques and tools just described, two flight-certified implementations of the models have been created and are being used in Chandra operations. One models the temperature of the power supply for the ACIS science instrument [ACIS]. The other models five temperatures on the Sun-pointed side of the forward structure that surrounds the X-ray mirror. Each week, as the schedule of observations for the following week is assembled the models are used to confirm that no thermal limits are violated. Separate cron jobs also run daily to perform post-facto "predictions" of temperatures for the previous three weeks. These are compared to actual telemetry and provide warning if the spacecraft behavior is drifting away from the existing model calibration.

\section{Summary}

The current Python ecosystem provides a strong platform for production science and engineering analysis. This paper discussed the specific case of developing thermal models for subsystems of the Chandra X-ray Observatory satellite. These models are now being used as part of the flight operations process.

In addition to the core tools (NumPy, IPython, Matplotlib, SciPy) that get used nearly every day in the author's work, two additional packages were discussed:

- Pytables / HDF5 is an easy way to handle the very large tables that are becoming more common in science analysis (particularly astronomy). It is simple to install and use and brings high performance to scientists.

- MPI for Python (mpi4py) with the MPICH2 library provides an accessible mechanism for parallelization of compute-intensive tasks.

\section{Acknowledgments}

Thanks to the reviewer James Turner for a detailed evaluation and helpful comments.

\section{REFERENCES}

[ACIS] http://cxc.harvard.edu/proposer/POG/html/ACIS.html

[CHANDRA] http://chandra.harvard.edu/

[FITS] http://fits.gsfc.nasa.gov/

[HDF5] http://www.hdfgroup.org/HDF5/

[MPI4PY] http://mpi4py.scipy.org/

[MPICH2] http://www.mcs.anl.gov/research/projects/mpich2/

[PYT] http://www.pytables.org

[SHP] http://cxc.harvard.edu/contrib/sherpa 